\begin{tabular}{|c|c|}
\hline Title & Powder X-ray diffraction observations of ice crystals formed from disaccharide solutions \\
\hline Author(s) & Uchida, T sutomu; Takeya, Satoshi \\
\hline Citation & $\begin{array}{l}\text { Physical Chemistry Chemical Physics, 12(45), 15034-15039 } \\
\text { https://doi.org/10.1039/c0cp01059f }\end{array}$ \\
\hline Issue Date & 2010-12-07 \\
\hline Doc URL & http:/hdl .handle.net/2115/47129 \\
\hline Rights & $\begin{array}{l}\text { Phys. Chem. Chem. Phys., 2010, 12, 15034 } 15039 \\
\text { - Reproduced by permission of the PCCPP Owner Societies }\end{array}$ \\
\hline Type & article (author version) \\
\hline File Information & PCCP12-45_15034-15039.pdf \\
\hline
\end{tabular}

Instructions for use 


\section{Powder X-ray diffraction observations of ice crystals formed from disaccharide solutions}

Tsutomu Uchida* $*^{a}$, Satoshi Takeya ${ }^{b}$

${ }^{a}$ Department of Applied Physics, Faculty of Engineering, Hokkaido University, N13 W8 Kita-ku, Sapporo 060-8628, Japan.

* E-mail: t-uchida@eng.hokudai.ac.jp; Fax: +81-11-706-6635; Tel: +81-11-706-6635

${ }^{b}$ Research Institute of Instrumentation Frontiers, National Institute of Advanced Industrial Science and Technology (AIST), Central 5, Higashi 1-1-1, Tsukuba 305-8565, Japan

\section{Summary}

Powder X-ray diffraction (PXRD) measurements on rapid freezing samples of disaccharide (trehalose, sucrose, and maltose) solutions indicated that the crystalline phases in the sample were both hexagonal and cubic ice. The cubic ice existed at a higher ratio in the higher disaccharide concentration samples. The temperature ramping experiments revealed that the cubic ice was stable below $233 \mathrm{~K}$, which was obviously higher than the temperature expected for a pure water system. The diffraction peak width of the hexagonal ice crystal was independent in the disaccharide concentrations. This indicated that the crystallite size of the hexagonal ice was more than several hundreds of nanometer, which coincided with the ice particle size previously observed in the freeze-fractured replica samples. The comparison of the present PXRD data with the replica observations by transmission electron microscope in an earlier study allows us to conclude that the cubic ice was formed at the grain boundary between the hexagonal ice and the coexisted non-crystalline disaccharide phase. (160 words)

Key words:

Powder X-ray diffraction, trehalose, sucrose, maltose, cubic ice

\section{Introduction}

Numerous studies have been undertaken to preserve living bodies and foods from freezing by the addition of cryoprotecting materials, such as natural substances (e.g., sugars and proteins) and synthetic chemicals (e.g., glycerin and dimethyl sulfoxide). Trehalose and sucrose in aqueous solutions are known to protect living species 
from damage caused by freezing. ${ }^{1}$ Trehalose is used as a cryoprotectant mainly for animals capable of enduring cold temperatures, whereas sucrose exists in plants. ${ }^{2}$ These natural cryoprotecting materials (disaccharides) are also used in medical and engineering applications, such as the cryopreservation of living cells. ${ }^{3}$ The cryoprotecting mechanisms of these materials are still not well understood, but their effects on the formation and stability of crystalline phases are important to study.

Sei et al. ${ }^{4}$ and Gonda and Sei ${ }^{5}$ measured the changes in single-crystal shapes and the crystal growth rates for ice in trehalose and sucrose solutions. They suggested that the cryopreservation mechanism of disaccharide solutions appeared to be a local increase in the viscosity near a growing ice interface, which impeded the ice-crystal growth. For a given temperature and the solute concentration, they found that trehalose reduced the growth rate more than sucrose. One of the reasons for the high inhibition effect of trehalose on ice growth is the expected formation of giant hydration clusters of trehalose in the solution ${ }^{5-7}$, since the transport properties were not so different from other disaccharide solutions. ${ }^{6-14}$

The freeze-fractured surfaces of the trehalose solution droplets were investigated by observing the replica films via a field-emission gun type transmission electron microscope (FEG-TEM). ${ }^{15}$ The FEG-TEM observations showed three typical textures on the trehalose solution replicas (see Fig. 1): (S) smooth surface, (P) fine particles (average diameter was $20-30 \mathrm{~nm}$ ), and (R) remaining part of the boundary between the smooth-surface area co-existing with the fine particles. The systematic observations indicated that the size of the smooth surface decreased exponentially as the trehalose concentration increased, whereas the number concentration of such area increased exponentially. Based on these observations, the smooth surface was predicted to be ice crystals, the fine particles were the precipitation of disaccharide molecules, and the remaining part was the glass state of the solutions. These fine particles may correspond to the giant hydration clusters of the trehalose predicted by previous studies. ${ }^{5-7}$ The confocal micro Raman spectroscopy revealed the similar textures of the ice crystals and the fine particles in the frozen solution composed of lysozyme and trehalose, ${ }^{\mathbf{1 6}}$ even though this technique has less spacial resolution than FEG-TEM. However, since the replica observation provides only information on the texture changes, the phase determination studies are required to understand the processes of the phase separation ${ }^{17}$ and the giant cluster formation..$^{5-7,15}$

In the present study, frozen samples of disaccharide solutions, which were formed using a similar method to the previous FEG-TEM observations, were measured by the powder X-ray diffraction (PXRD) technique to understand the phase separations and phase changes of frozen disaccharide solutions. 


\section{Experimental}

We focused primarily on trehalose, which has been well studied by the FEG-TEM observations. ${ }^{15}$ Research-grade trehalose dihydrate crystals were donated by Hayashibara Biochemical Laboratories, Inc. To compare the properties observed in the trehalose solutions, we also conducted investigations using sucrose (of the highest quality; Sigma-Aldrich Japan, Inc.) and maltose (research grade, Hayashibara Biochemical Laboratories, Inc.). The purity of the distilled and deionized water used for the experiments was approximately $5 \times 10^{-6} \mathrm{~S} \mathrm{~m}^{-1}$ of conductivity.

The sample preparation procedures were almost the same as those of the previous study. ${ }^{15}$ We prepared the disaccharide (trehalose, sucrose, and maltose) solutions at concentrations ranging from $20 \mathrm{wt} \%$ to $50 \mathrm{wt} \%$ (Table 1). The concentration of the solutions was determined by measuring the masses of the disaccharides and pure water, taking into consideration the hydrated water. The accuracy of the concentration was estimated to be $\pm 0.1 \mathrm{wt} \%$. These solutions (approximately $1 \mathrm{mg}$ ) were then rapidly frozen in a liquid nitrogen atmosphere (approximately $80 \mathrm{~K}$ ) with a freezing rate of approximately $3 \times 10^{2} \mathrm{~K} \mathrm{~min}^{-1}$. The order of this value was almost the same (ranging within $\pm 50 \mathrm{~K} \mathrm{~min}^{-1}$ ) in all disaccharide solutions, even though the solutions were relatively high concentrations. To investigate the effects of the freezing rate on the crystallography of the respective samples, we also observed samples prepared at slower freezing rates. A slow freezing-rate sample was prepared for each highest concentration sample by storing it in a refrigerator at a relatively high temperature of approximately $255 \mathrm{~K}$ (freezing rate: approximately $1 \mathrm{~K} \mathrm{~min}^{-1}$ ).

These frozen samples were finely powdered in a nitrogen atmosphere at a temperature below $100 \mathrm{~K}$. The fine-powdered samples were top-loaded on a specimen holder made of $\mathrm{Cu}$ and mounted onto the powder X-ray diffractometer (Rigaku, type Ultima III). The PXRD measurements were done at $93 \mathrm{~K}$ in the $\theta / 2 \theta$ step scan mode with a step width of 0.02 degree in the $2 \theta$ range of $6^{\circ}$ to $43^{\circ}$ using the $\mathrm{CuK} \alpha$ radiation $(40 \mathrm{kV}, 40 \mathrm{~mA})$.

To investigate the stability of the crystalline phases, we also performed temperature ramping PXRD measurements in the $2 \theta$ range of $22^{\circ}$ to $27^{\circ}$ on the highest concentration sample of each disaccharide solution. The sample temperature was increased from $153 \mathrm{~K}$ to $273 \mathrm{~K}$ with a temperature increase rate of approximately $1 \mathrm{~K}$ $\min ^{-1}$ and maintained at a measurement temperature within $\pm 1 \mathrm{~K}$ for each 200 sec for PXRD measurement.

\section{Results and discussion}




\section{Crystalline structures of frozen disaccharide solutions}

The PXRD profiles of various concentrations of trehalose solutions frozen using the rapid process were shown in Fig. 2. In the $23 \mathrm{wt} \%$ trehalose sample, typical diffraction pattern for hexagonal ice Ih (space group $P 6_{3} / m m c$ ) was observed. However, as the concentration of trehalose increased, background intensity in the $2 \theta$ range of $22^{\circ}$ to $27^{\circ}$ increased, and the relative peak intensity ratios of the ice $\operatorname{Ih}(110)$ and ice $\operatorname{Ih}(002)$ reflections changed since ice Ih(002) reflection overlaps with those of cubic ice Ic(111). This result suggests that the sample included some amount of ice Ic in a high concentration sample, and the volume ratio of ice Ic to Ih increased as the concentration of trehalose increased. This is the first evidence of ice Ic existence in the frozen sample of the disaccharide solutions. In both the sucrose and maltose solutions (see Figs. 3a and b), the ice Ic phase was observed in all the disaccharide solutions especially at higher concentrations as well as in trehalose.

On the other hand, the PXRD profile on the slowly frozen trehalose solution sample of $47 \mathrm{wt} \%$ shows two typical phases of ice Ih and trehalose dihydrate crystals ${ }^{18}$ (Fig. 4). For the highest concentration samples of sucrose and maltose solutions, neither ice Ic nor their crystal diffraction peaks were observed. Therefore, it is concluded that ice Ic crystal is formed in higher disaccharide solutions when a small amount of their solutions were quenched at the liquid nitrogen temperature. Additionally, trehalose seems to easily form dihydrate crystals in comparison to the cases of sucrose and maltose.

\section{Temperature ramping measurements of frozen samples}

For the frozen samples of the highest disaccharide concentration solutions quenched in liquid nitrogen, the temperature ramping PXRD measurements were performed every $10 \mathrm{~K}$ from $93 \mathrm{~K}$ to $273 \mathrm{~K}$. The initial profile of the $47 \mathrm{wt} \%$ trehalose solution at 93K (the farthest side profile of Fig. 5a) was similar to those in Figure 2, which was closed up in the $2 \theta$ range of $22^{\circ}$ to $27^{\circ}$. Three large peaks with a high background indicated that this sample included both ice Ih and ice Ic crystals. The PXRD profile did not change during the temperature ramping experiment up to $233 \mathrm{~K}$, but the profile drastically changed at $243 \mathrm{~K}$; the three large diffraction peaks became sharpened and the base line decreased down to the noise level. These results indicate that the ice Ic phase disappeared below $243 \mathrm{~K}$ whereas the ice Ih still remained even above $243 \mathrm{~K}$. At $273 \mathrm{~K}$, the whole ice Ih signals disappeared due to its melting. Instead, the trehalose dihydrate crystal was formed simultaneously.

A similar stability condition of the ice Ic crystal was also observed in the sucrose (Fig. 5b) and maltose (Fig. 5c) solutions. The transition temperature from ice Ic to ice Ih in both samples was also observed between 233 and $243 \mathrm{~K}$. These two samples showed, however, no crystal formation at $273 \mathrm{~K}$, that is, all solid phases were 
melted at the ice melting point. These results suggest that trehalose forms its dihydrate crystal at temperatures above the ice melting point, whereas sucrose and maltose didn't form their crystals at the same temperature.

\section{Formation and stability of cubic ice phase in frozen disaccharide solutions}

As mentioned above, the PXRD measurements on the rapid freezing samples of the disaccharide solutions at the liquid nitrogen temperature revealed that the crystalline phase was either ice Ih or ice Ic. The ice Ic phase was stable up to $233 \mathrm{~K}$, which is higher than expected from previous phase transition temperature on the vapor deposit ice Ic $^{19}$ but similar to that observed in the glycerol solution, ${ }^{20-21}$ in the glucose solution, ${ }^{22-23}$ or in the ionic salt solutions. $^{24}$

In a pure water system, ice Ic can be obtained by the following methods: 1) from the high-pressure phase of ice (ice III or VII) by a decrease of the pressure during an increase of temperature, ${ }^{25}$ 2) from the temperature increase of amorphous ice condensed on a cold surface (below $110 \mathrm{~K}$ ), ${ }^{19}$ or 3) the quenching of a water droplet at a rate of $10^{4} \mathrm{~K} \mathrm{~s}^{-1} \cdot{ }^{26-29}$ The ice Ic phase is metastable and it transforms to the stable ice Ih phase when it is warmed up above approximately $143 \mathrm{~K}$ and the reverse transformation is never observed.

However, the crystallographic observations of the frozen samples of the glycerol solution, ${ }^{20-21}$ glucose solution, ${ }^{22-23}$ and ionic salt solutions ${ }^{24}$ have revealed that ice Ic was formed in them. Vigier et al. ${ }^{20}$ estimated the average diameter of ice Ic in the frozen glycerol solutions to be 5 to $15 \mathrm{~nm}$. The ice Ic phase formed by quenching of the aqueous solutions mentioned above transformed to ice Ih above $200 \mathrm{~K}$, which was obviously higher than that observed in the pure water system. This process is considered to occur naturally, that is, small droplets of water in the upper atmosphere may often freeze directly into ice Ic. ${ }^{\mathbf{3 0 - 3 4}}$ Mayer and Hallbrucker ${ }^{\mathbf{2 8}}$ demonstrated that ice Ic formed in the quenched aqueous aerosol droplets and that it was stable up to approximately $200 \mathrm{~K}$. Other earlier studies had revealed that the ice Ic formed in confined space. ${ }^{35-39}$ Takamuku et al..$^{35}$ reported that ice Ic was formed from water existed in a porous silica of $3 \mathrm{~nm}$ and $10 \mathrm{~nm}$ in diameter. It was found that the ice Ic formed in the confined space was also more stable than that in the pure water system.

Taking into account the earlier studies reported, the formation of ice Ic in disaccharide solutions is expected to occur in a confined meso-pore space. In general, the full-width of the half maximum intensity of the $\mathrm{x}$-ray diffraction peaks expand ${ }^{40}$ when the crystallite size is several hundred nano-meters or less. However, the diffraction peaks of ice Ih in the present study were not broadened even in the highest concentration sample. Therefore, it is considered that the formation sites of ice Ih and ice Ic were different, and the crystallite size of ice Ih would be ranged from micron to sub-micron order. 


\section{Comparison of PXRD results with FEG-TEM image of replica samples}

Earlier studies of the freeze-fractured replica observation via FEG-TEM showed three typical features ${ }^{15}$ (see Figure 1): (S) smooth surface as the ice crystal, (P) fine particles as the precipitation of disaccharide molecules, and $(\mathrm{R})$ the remaining part as the glass state of the solutions. Here, we compare the PXRD results with the FEG-TEM observations reported from the crystallographic point of view.

The smooth surface as ice crystal observed by FEG-TEM would be identified not to be ice Ic but ice Ih crystal because the size of the smooth surface was several micron, as discussed in the previous section.

The fine particles were considered to be the precipitation disaccharide molecules by the viscosity measurement, ${ }^{7}$ but their size of 20 - $30 \mathrm{~nm}$ in diameter was similar to those expected for the ice Ic crystal. ${ }^{20}$ On the other hand, by the temperature ramping experiments with FEG-TEM, all fine particles disappeared whereas the other textures remained similar to the $90 \mathrm{~K}$ sample during the annealing at $150 \mathrm{~K}$. All crystalline phases kept their original crystal structure as it is formed in the quenching process because no change was observed in the PXRD profiles at the annealing temperature of $150 \mathrm{~K}$. Thus it is suggested that the fine particles observed by the FEG-TEM were not the crystalline ice Ic phase. This would support the prediction ${ }^{15}$ that the fine particles were related to the disaccharide concentrations. Based on the PXRD pattern, no crystalline disaccharide existed at approximately $150 \mathrm{~K}$ when the sample was frozen rapidly. Therefore, the fine particles should be an amorphous agglomeration of the disaccharide molecules.

After annealing at $240 \mathrm{~K}$, the FEG-TEM observations show that the edge of the smooth surface had a slightly larger contrast and the remaining part (R) was dug deeply. ${ }^{15}$ These texture changes suggest that the phase of the remaining part was changed at around $240 \mathrm{~K}$. The PXRD measurements in the present study suggest that the ice Ic phase disappeared between $233 \mathrm{~K}$ and $243 \mathrm{~K}$, and that the only crystalline phase that remained was the ice Ih. The consistency of these temperatures, at which the texture change and the diffraction pattern change, suggests that the ice Ic phase existed in the third texture, where the remaining part (R) was observed in the FEG-TEM image. Therefore, the ice Ic crystals didn’t have characteristic textures in the replica image, and the ice Ic existed in a different part of the ice Ih phase. This is consistent with the FEG-TEM observations that the area of the smooth surface decreased whereas the fine particles and the remaining part increased at a higher disaccharide concentration range.

Uchida et al. ${ }^{15}$ reported that there were no fine particles in the slow freezing rate samples of the trehalose solution. They considered that all the concentrated solute was out of the area of observation. However, 
the present study shows that the slow freezing rate sample of the trehalose solutions includes not only the ice Ih but also the trehalose dihydrate crystal. Then we suggest that the FEG-TEM image of the freeze-fractured replica of the trehalose solution prepared at the slow freezing rate may include both ice Ih and trehalose dihydrate crystals, even though it is difficult to distinguish them on the freeze-fractured replica image by FEG-TEM.

\section{Formation mechanism of two different ice phases}

Based on the phase determination observed in the freeze-fractured replica samples by FEG-TEM, the ice Ic with less than a hundred nanometer in size was formed at the grain boundary between the ice Ih phase, and the disaccharide precipitated fine particles were formed within the grain boundary. The ice Ic would co-exist with the disaccharide precipitated particles, which may correspond to the giant disaccharide clusters. ${ }^{5-7,15}$ Moreover, the FEG-TEM images on the replica annealed at $150 \mathrm{~K}$ indicated that the fine particles disappeared whereas no profile changes in the PXRD were observed. If the ice Ic phase was formed on the fine particles, the ice Ic should be changed into the stable state at that moment. Thus we consider that the ice Ic would be formed between the disaccharide clusters by freezing of free water molecules, which locate around disaccharide molecules but are independent of the disaccharide clusters or glass phase.

The temperature ramping PXRD measurements on the rapid frozen sample of the highest concentration solutions indicated that the ice Ic phase was stable up to $233 \mathrm{~K}$, which is much higher than that expected from the pure water system (approximately $143 \mathrm{~K}^{\mathbf{1 9}}$ ). This anomalous stability has been reported in other solution systems (including glycerol, ${ }^{20-21}$ glucose, ${ }^{22-23}$ salts, ${ }^{24}$ or aqueous aerosol ${ }^{28}$ ), or in the confined space systems (in porous silica $^{35}$ or in emulsion ${ }^{39}$ ). Then the ice Ic phase is considered to be stabilized by foreign molecules. In the disaccharide solution system, the present study suggests that ice Ic exists between the disaccharide clusters. Then the water molecules constructing the ice Ic would slightly interact with disaccharide molecules even though they are not bound to the disaccharide molecules. The transition temperature from ice Ic to ice Ih at around $240 \mathrm{~K}$ which coincides well with the glass-transition temperature ( $\mathrm{Tg}$ ') of the disaccharide solution; $\mathrm{Tg}$ ' was approximately $238 \mathrm{~K}, 234 \mathrm{~K}$ and $233 \mathrm{~K}$ for the $50 \mathrm{wt} \%$ concentration of the trehalose, sucrose and maltose solutions, respectively. ${ }^{9}$ This is also the case for the Tg' of glucose $(230 \mathrm{~K})^{22-23}$ and the eutectic point of $67 \mathrm{wt} \%$ glycerol $(230 \mathrm{~K}){ }^{20-21}$ These coincidence allow us to consider that the transition of ice Ic to ice Ih is difficult to occur spontaneously in frozen solution systems because the foreign molecules might stabilize the system, but is induced by the high mobility of the foreign molecules. Therefore the transition of ice Ic to ice Ih observed in the present study may occur with the devitrification of the disaccharide molecules. 
At a slow freezing rate of the trehalose solution, ice Ic was not formed, instead the trehalose dihydrate crystal was formed. Based on the temperature ramping measurements, the trehalose dihydrate crystal was formed at temperatures above the ice melting point. Then the slow freezing of the trehalose solution would lead to the formation of the macroscopic crystals, trehalose dihydrate and ice Ih, instead of the glassy phase or the microscopic crystals of ice Ic. Trehalose has the lowest aqueous solubility in the three disaccharides, ${ }^{41-42}$ so trehalose is the easiest material to form the hydrated crystal during the freezing of the three disaccharide solutions. The formation of the dihydrate crystal would be a disadvantage for the cryopreservant since it may result in the mechanical stress or the osmotic stress in living bodies. Therefore, the trehalose may act as the cryoprotectant especially in rapid freezing process as is observed in the cryoprotecting study on living neuronal cells in the solutions including trehalose. ${ }^{3}$

Based on the PXRD measurements, three typical textures observed in the disaccharide solution replicas were identified, and the phase separation process during the freezing of the solutions was understood. When a small amount of solution was quenched at the liquid nitrogen temperature, the disaccharide molecules tend to form the giant clusters which were observed as fine particles in the replica samples. On the other hand, the free water molecules apart from the disaccharide molecules form ice Ih crystals. The crystallite size of the ice Ih is larger than the sub-micron order. Then ice Ih crystal is determined as the smooth surface area in the replica samples. The intermediate region of these phases is constructed by water molecules which are located in a confined meso-pore space between the giant clusters and slightly interact with the disaccharide molecules. These water molecules form the metastable ice Ic crystal (Fig. 6). When the disaccharide concentration is high, a large amount of confined space would be formed between the giant clusters. Then the total amount of ice Ic was larger in the higher disaccharide concentration samples. Since these ice Ic crystals were anomalously stable up to $233 \mathrm{~K}$, the confined space would be maintained until the disaccharide molecules change their molecular network at their devitrification temperatures.

\section{Conclusions}

The powder X-ray diffraction measurements on the freezing samples of the trehalose, sucrose and maltose solutions were carried out to determine the microscopic distributions of the water and disaccharide molecules, which had been investigated by the freeze-fractured replica observation via a field-emission gun type transmission electron microscope. ${ }^{15}$ 
By the rapid freezing of disaccharide solutions (concentration ranged from $20 \mathrm{wt} \%$ to $50 \mathrm{wt} \%$ ), the sample included both hexagonal and cubic ice crystals. This is the first report of cubic ice formation from disaccharide solutions, which was consistent with the results obtained in the glycerol, ${ }^{20-21}$ glucose, ${ }^{22-23}$ ionic salts ${ }^{24}$ and aqueous aerosol. ${ }^{28}$ On the other hand, the slow freezing process only formed the hexagonal ice crystal, except for the formation of the trehalose dihydrate crystal in the highest concentration solution. The temperature ramping measurements on the rapid freezing samples revealed that no diffraction pattern change was observed at temperatures between 93 and $233 \mathrm{~K}$. All cubic ice phases disappeared in the three disaccharide samples at $243 \mathrm{~K}$, which was a much higher temperature than that expected from the cubic ice formed in the pure water system.

These results were compared with the textures observation of freeze-fractured replica samples by FEG-TEM. ${ }^{15}$ The smooth surface expected as the ice phase was determined to be the hexagonal ice crystals because the mass fraction and the particle size estimations changed with the disaccharide concentrations. The fine particles were concluded to be the non-crystalline phase, so they would be the agglomerations of the disaccharide molecules. Then the cubic ice phase had no characteristic texture in the replica samples. Based on the coincidence of the transition temperature from the cubic ice to the hexagonal ice with the glass transition temperature of the disaccharide solutions, we considered that the cubic ice existed at the grain boundary between the hexagonal ice and other glass state of the disaccharide molecules. This coincidence also suggested that the phase transition from the cubic ice to the hexagonal ice was difficult to occur spontaneously in the frozen aqueous solution system but was induced by the high mobility of the disaccharide molecules. In conclusion, the water molecules were considered to be separated into three phases during the rapid freezing process of the disaccharide solutions: 1) the disaccharide binding water molecules didn't contribute to the crystallization, 2) the free water with little interaction with the disaccharide molecules would form hexagonal ice, and 3) the intermediate free water, which interacted with the disaccharide molecules but didn't bind with them, would form cubic ice in the confined space between the disaccharide giant clusters.

Compared to the rapid freezing process, the slow freezing process indicated no cubic ice formation. Trehalose was found to be the easiest molecules to form the hydrated crystal in the three disaccharide molecules. Accordingly, the cryoprotecting effect of the trehalose would be great in the rapid freezing process.

\section{Acknowledgements}

Part of this work was supported financially by the Society for Techno-innovation of Agriculture, Forestry and Fishers. 


\section{References}

1 T. Furuki, M. Sakurai, Cryobio. Cryotech. 2005, 51, 1 (in Japanese with English abstract).

2 J. H.Crowe, L. M. Crowe, J. F. Carpenter, A. S. Rudolph, C. A. Wistrom, B. J. Spargo, T. J. Anchrodoguy, Biochim. Biophys. Acta, 1988, 947367.

3 J. Motomura, T. Uchida, M. Nagayama, K. Gohara, T. Taira, K. Shimizu, M. Sakai, in Physics and Chemistry of Ice, ed. W. F. Kuhs, Royal Society of Chemistry, London, 2007, pp. 409.

4 K. Sei, T. Gonda, Y. Arima, J. Cryst. Growth, 2002, 240, 218.

5 T. Gonda, K. Sei, Progress in Crystal Growth and Characterization of Materials, 2005, 51, 70.

6 A. Lerbret, P. Bordat, F. Affouard, M. Descamps, F. Migliardo, J. Phys. Chem. B, 2005, 109, 11046.

7 T. Uchida, M. Nagayama, K. Gohara, J. Crys. Growth, 2009, 311, 4747.

8 S. Magazu, G. Maisano, P. Migliardo, E. Tettamanti, V. Villari, Mol. Phys., 1999, 96, 381.

9 C. Branca, S. Magazu, G. Maisano, F. Migliardo, P. Migliardo, G. Romeo, J. Phys. Chem. B, 2001, 105, 10140.

10 T. Matsuoka, T. Okada, K. Murai, S. Koda, H. Nomura, J. Mol. Liquids, 2002, 98-99, 317.

11 Y. Nagasawa, Y. Nakagawa, J. Kenmochi, T. Okada, Cryobio. Cryotech., 2003, 49, 87.

12 N. Ekdawi-Sever, J. J. de Pablo, E. Feick, E. von Meerwall, J. Phys. Chem. A, 2003, 107, 936.

13 M. P. Longinotti, H. R. Corti, J. Phys. Chem. Ref. Data, 2008, 37, 1503.

14 H. R. Corti, G. A. Frank, M. C. Marconi, J. Phys. Chem. B, 2008, 112, 12899.

15 T. Uchida, M. Nagayama, T. Shibayama, K. Gohara, J. Cryst.Growth, 2007, 299, 125.

16 J. Malsam, A. Aksan, J. Phys. Chem. B, 2009, 111, 6792.

17 J. Dong, A. Hubel, J. C. Bischof, A. Aksan, J. Phys. Chem. B, 2009, 113, 10081.

18 H. Nagase, N. Ogawa, T. Endo, M. Shiro, H. Ueda, M. Sakurai, J. Phys. Chem. B, 2008, 112, 9105.

19 L. G. Dewell, A. P. Rinfret, Nature, 1960, 188, 1144.

20 G. Vigier, G. Tholllet, R. Vassoille, J. Cryst. Growth, 1987, 84, 309.

21 V. Berejnov, N. S. Husseini, O. A. Alsaied, R. E. Thorne, J. Appl. Cryst., 2006, 39, 244.

22 K. Kajiwara, P. Thanatuksom, N. Murase, F. Franks, CryoLett., 2008, 29, 29.

23 P. Thanatuksora, K. Kajiwara, N. Murase, F. Franks, Phys. Chem. Chem. Phys., 2008, 10, 5452.

24 B. J. Murray, D. A. Knopf, A. Bertram, Nature, 2005, 434, 202.

25 P. V. Hobbs, P. V. Ice Physics, Clarendon. Oxford: Oxford, 1974. 
26 P. Bruggeller, E. Mayer, Nature, 1980, 288, 569.

27 J. Dubochet, A. W. McDowall, J. Microsc., 1981, 124, RP3.

28 E. Mayer, A. Hallbrucker, Nature, 1987, 325, 601.

29 G. P. Johari, J. Chem. Phys., 2005, 122, 194504-1.

30 T. Kobayashi, Y. Furukawa, T. Takahashi, J. Cryst. Growth, 1976, 35, 262.

31 E. Whalley, Science, 1981, 211, 389.

32 E. Whalley, J. Phys. Chem., 1983, 87, 4174.

33 T. Takahashi, T. Kobayashi, J. Cryst. Growth, 1983, 64, 593.

34 E. Whalley, G. E. McLaurin, J. Opt Soc. Am., 1984, A1, 1166.

35 T. Takamuku, M. Yamagami, H. Wakita, Y. Masuda, T. Yamaguchi, J. Phys. Chem. B, 1997, $101,5730$.

36 D. C. Steytler, J. C. Dore, C. J. Wright, J. Phys. Chem., 1983, 87, 2458.

37 H. K. Christenson, J. Phys.: Condens. Matter, 2001, 13, R95.

38 K. Morishige, H. Iwasaki, Langmuir, 2003, 19, 2808.

39 B. J. Murray, A. K. Bertram, Phys. Chem. Chem. Phys., 2006, 8, 186.

40 R. Jenkins, R. L. Snyder, in Introduction to X-ray powder diffractometry, Chemical analysis, ed. J. D. Winefordner, John Wiley \& Sons, Inc., New York, 1996, pp. 89.

41 T. Higashiyama, Pure Appl. Chem., 2002, 74, 1263.

42 D. R. Lide (ed.), CRC Handbook of Chemistry and Physics (84 ${ }^{\text {th }}$ ed.), CRC Press, Boca Raton, 2003 , pp. 8.

\section{Figure captions}

Table 1 Sample concentrations used for PXRD measurements. The same concentration number listed in a disaccharide means that we checked the reproducibility of the results.

Fig. 1 FEG-TEM image of the freeze-fractured replica prepared by quenching of the 35 wt $\%$ trehalose solution (Scale bar: $100 \mathrm{~nm}$ ). Three typical features are (S) smooth surface, (P) fine particles and (R) remaining part.

Fig. 2 PXRD patterns on rapid frozen samples of various concentrations of trehalose solutions. The short bars indicate the positions of the diffraction patterns for both hexagonal ice Ih and cubic ice Ic. 
Fig. 3 PXRD patterns on rapid frozen samples of (a) sucrose solutions (39 and $50 \mathrm{wt} \%$ in the original concentration) and (b) maltose solutions (35 and $47 \mathrm{wt} \%$ ).

Fig. 4 PXRD patterns on slowly frozen samples of (a) 47 wt $\%$ concentration of trehalose, (b) 50 wt\% concentration of sucrose, and (c) 50 and $47 \mathrm{wt} \%$ concentration of maltose solutions. The diffraction pattern of the trehalose sample shows that the frozen sample was mixture of ice Ih and the trehalose dihydrate crystal (simulated by the previous work ${ }^{18}$ for comparison). On the other hand, two samples included only ice Ih crystals.

Fig. 5 Series of PXRD patterns on quenched samples of (a) $47 \mathrm{wt} \%$ concentrations of trehalose, (b) $50 \mathrm{wt} \%$ concentrations of sucrose, and (c) $47 \mathrm{wt} \%$ concentrations of maltose solutions with a temperature ramping from $93 \mathrm{~K}$ to $273 \mathrm{~K}$ in each $10 \mathrm{~K}$.

Fig. 6 Phase separation image during the freezing of the disaccharide solutions. 


\begin{tabular}{ll} 
Disaccharide & Concentration $[\mathrm{wt} \%]$ \\
Trehalose & $23,25,34,39,44,44,46,47$ \\
Sucrose & $33,39,39,50$ \\
Maltose & $27,35,39,47$ \\
\hline
\end{tabular}


Graphic contents entry

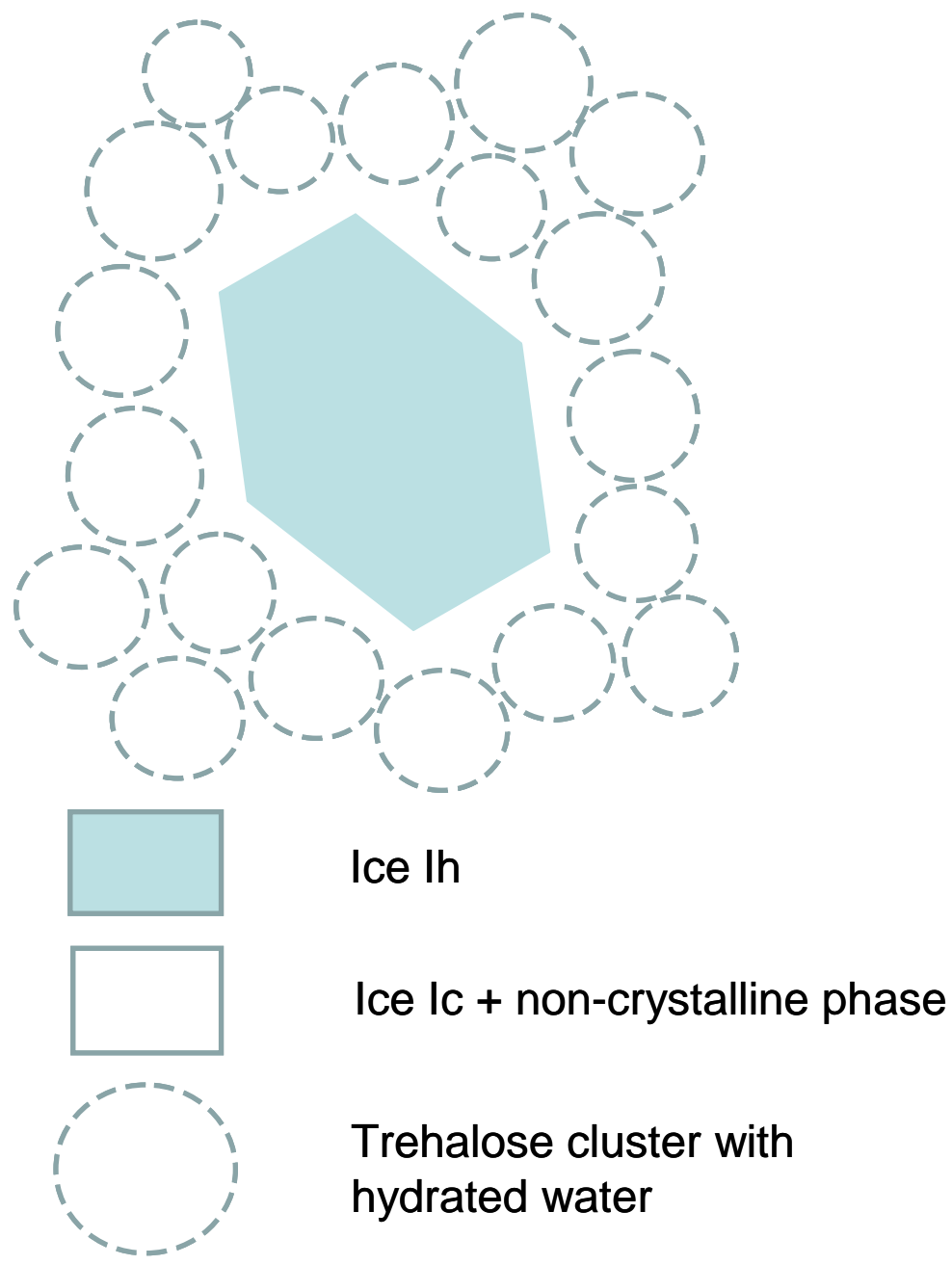

Powder X-ray diffraction on the particles of the disaccharide (trehalose, sucrose or maltose) solution quenched in liquid nitrogen revealed that the sample included not only the stable hexagonal ice phase but also the metastable cubic ice which was anomalously existed up to $233 \mathrm{~K}$. 


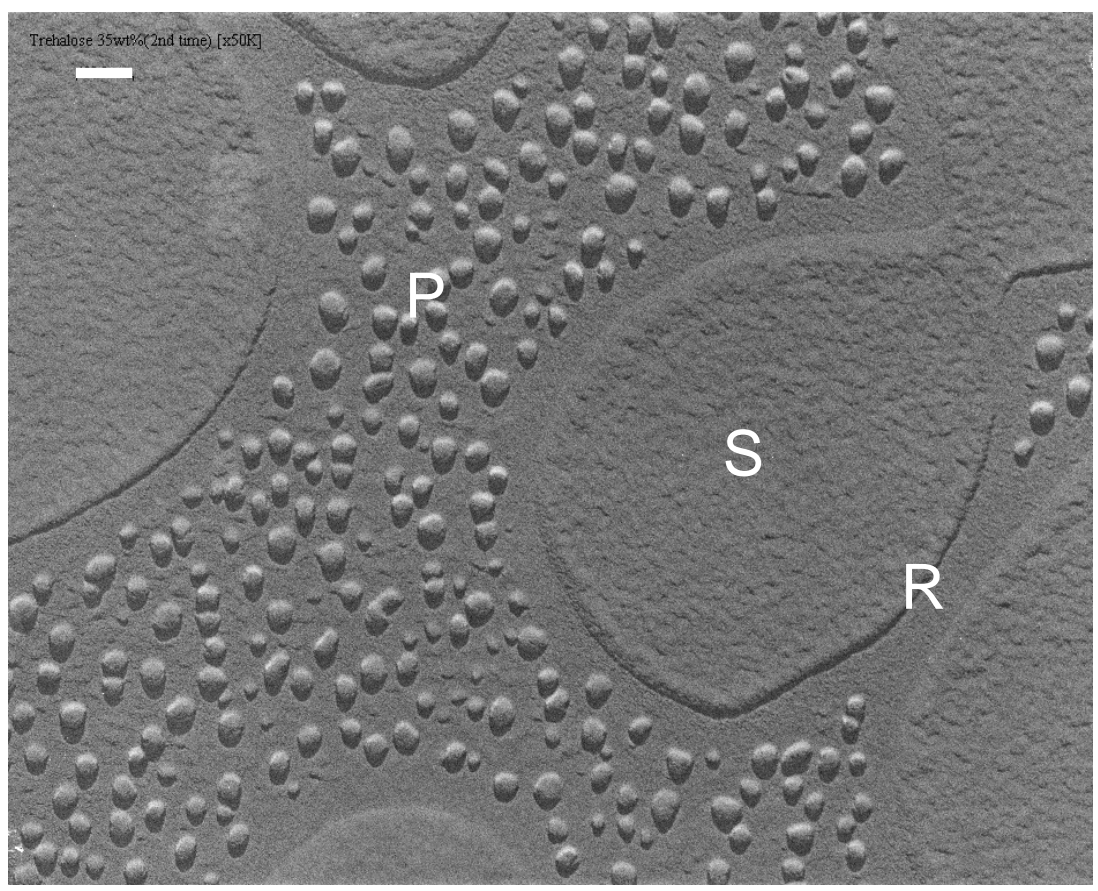

Fig. 1 


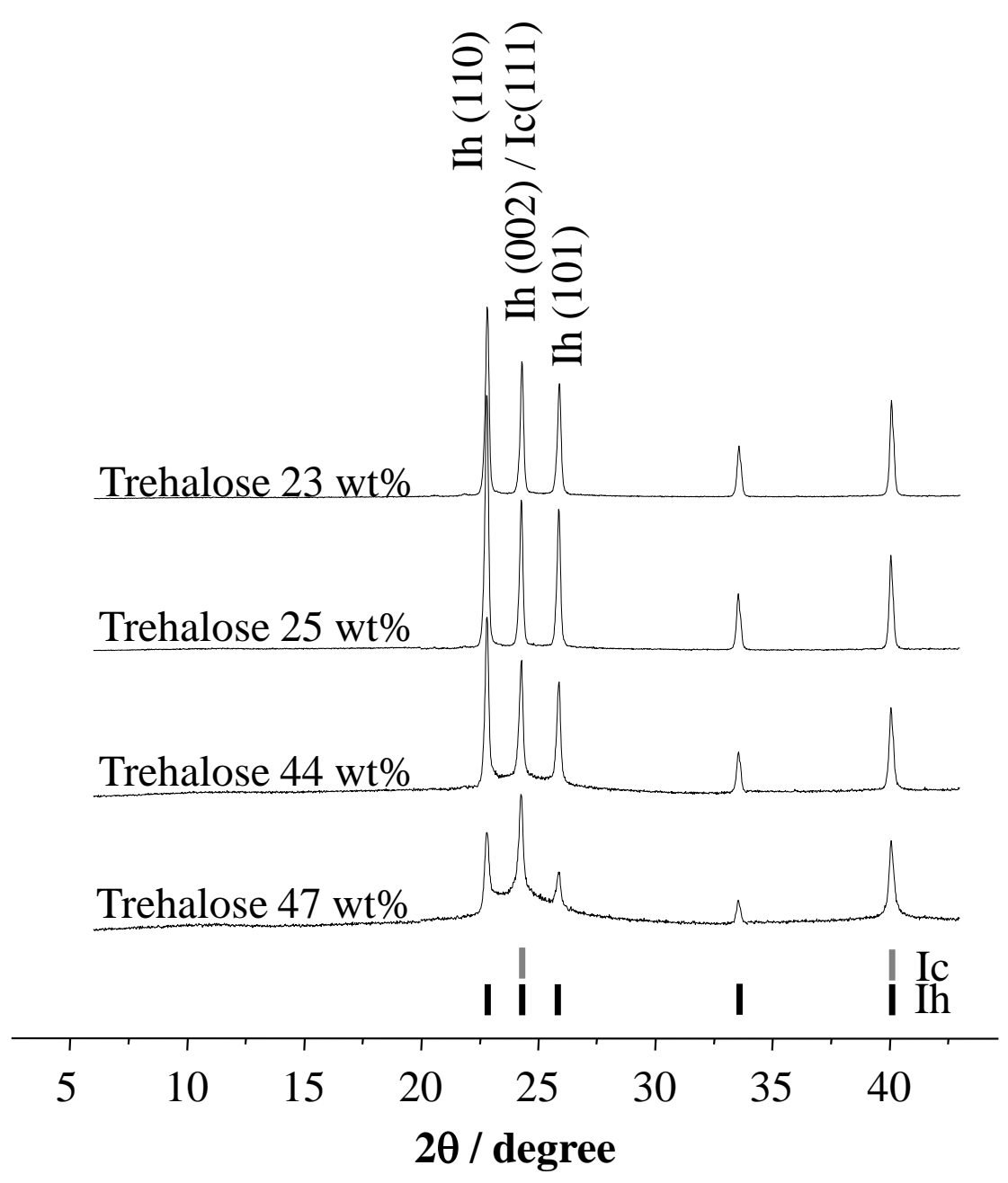

Fig. 2 


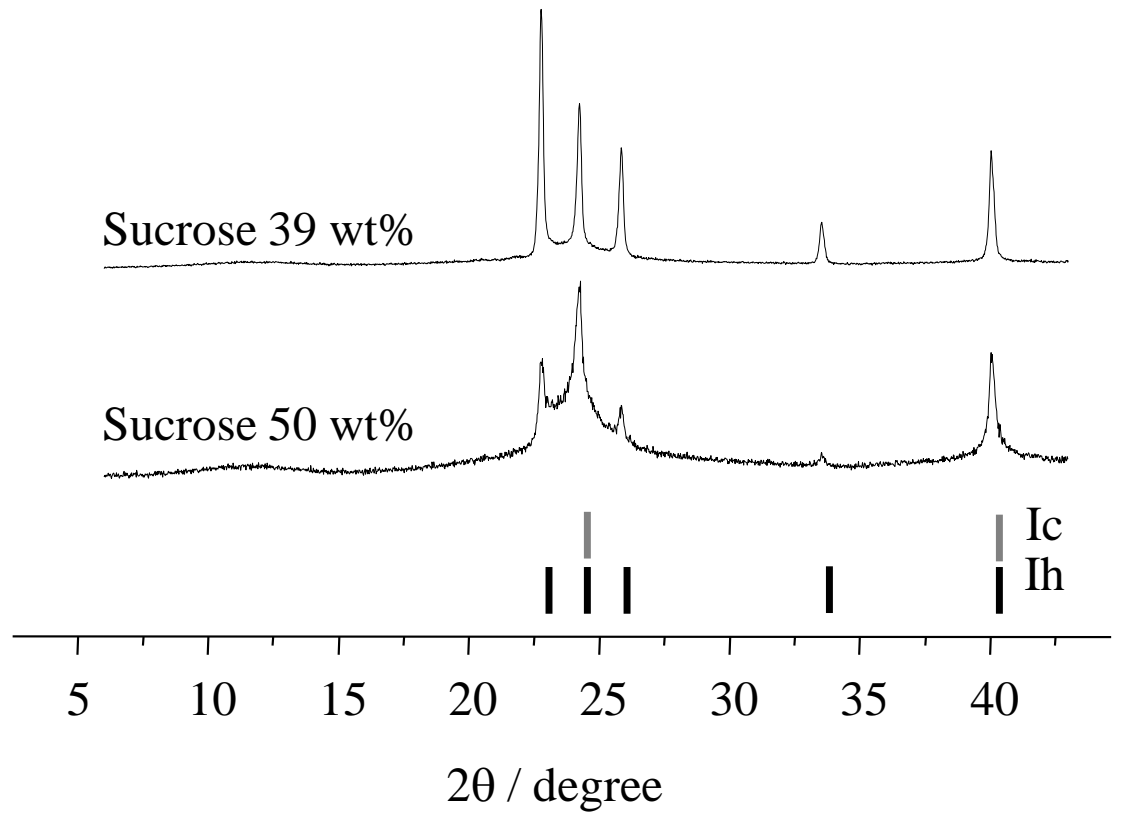

Fig. 3a 


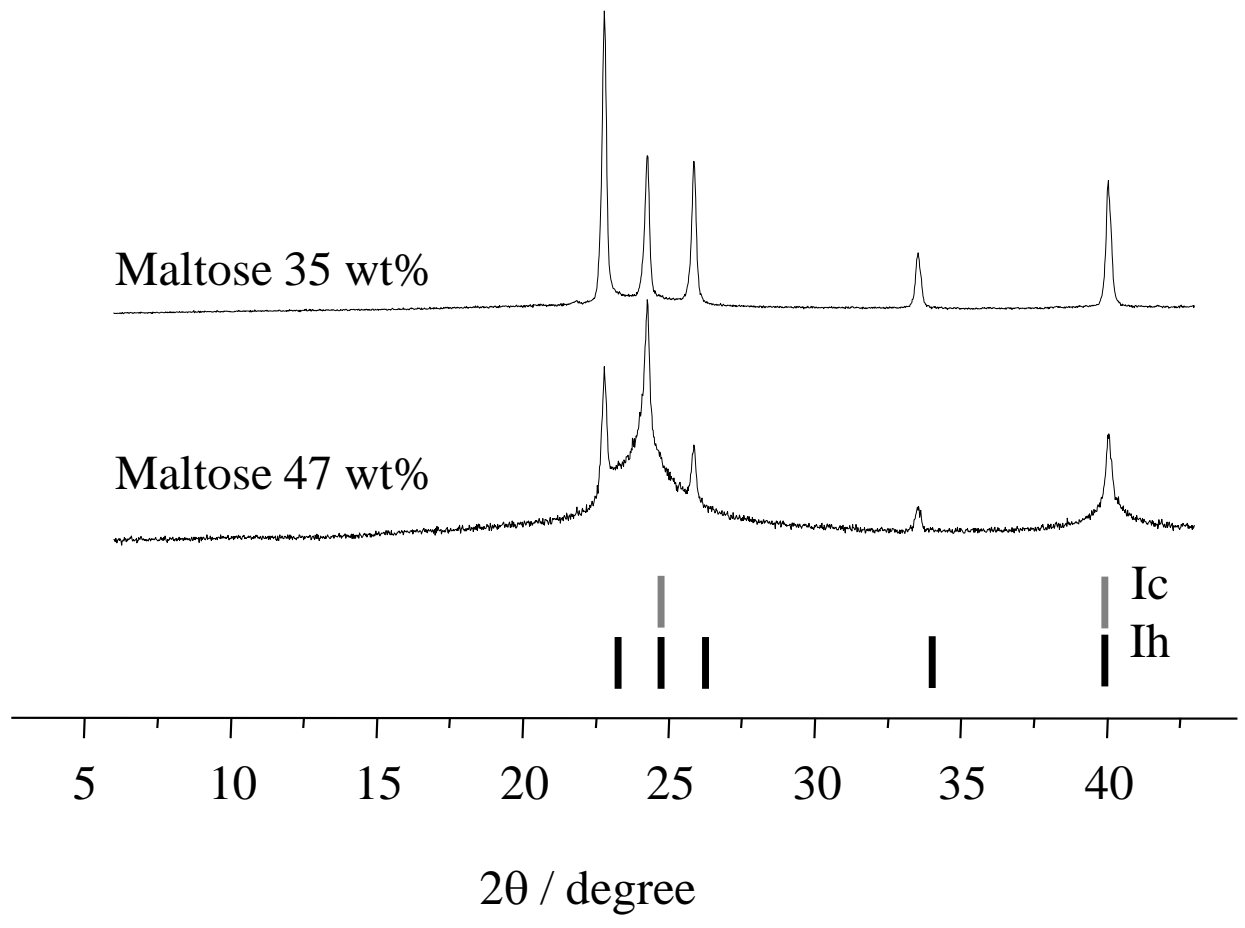

Fig. 3b 
c) Maltose $47 \mathrm{wt} \%$

b) Sucrose $50 \mathrm{wt} \%$

a) Trehalose $47 \mathrm{wt} \%$
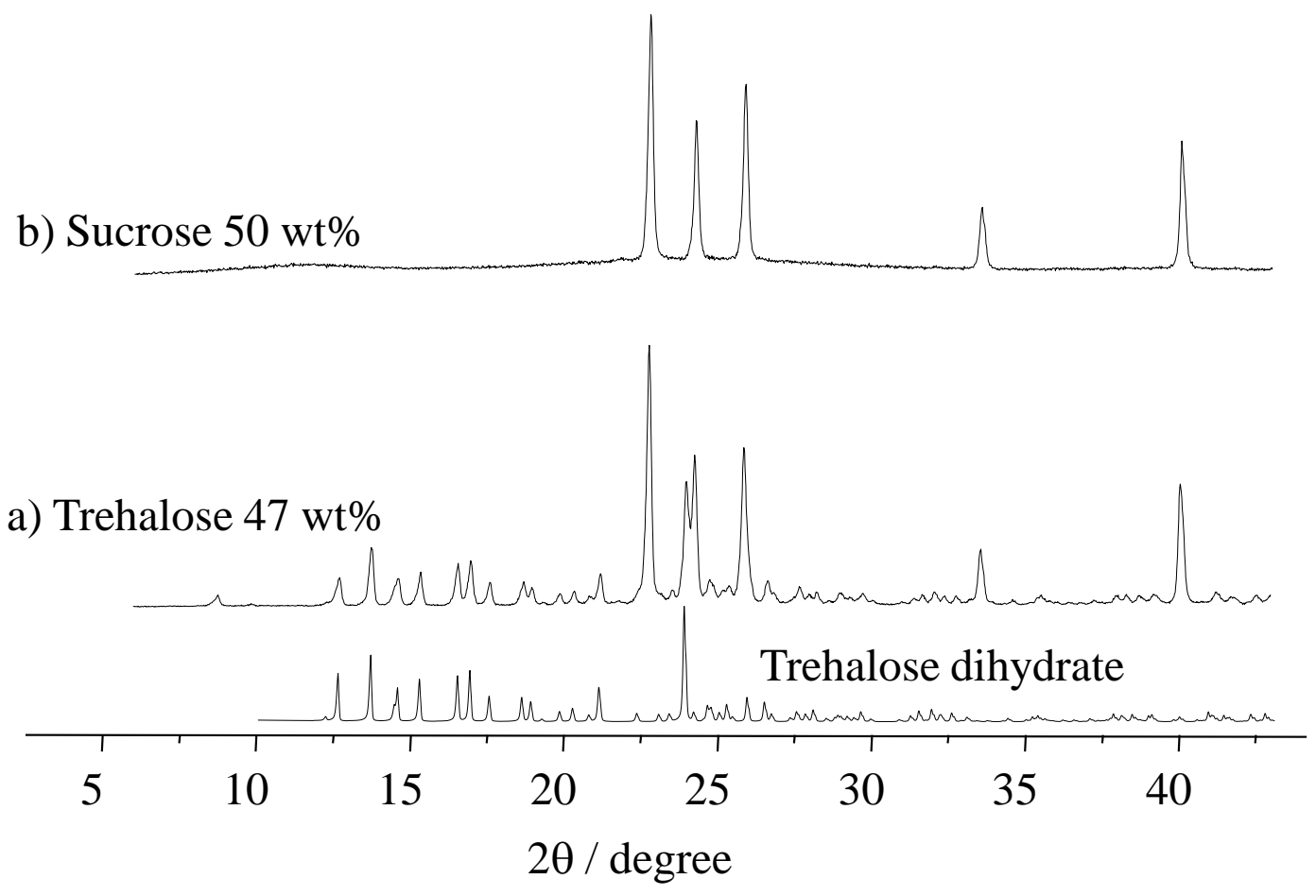

Fig. 4 
(a)

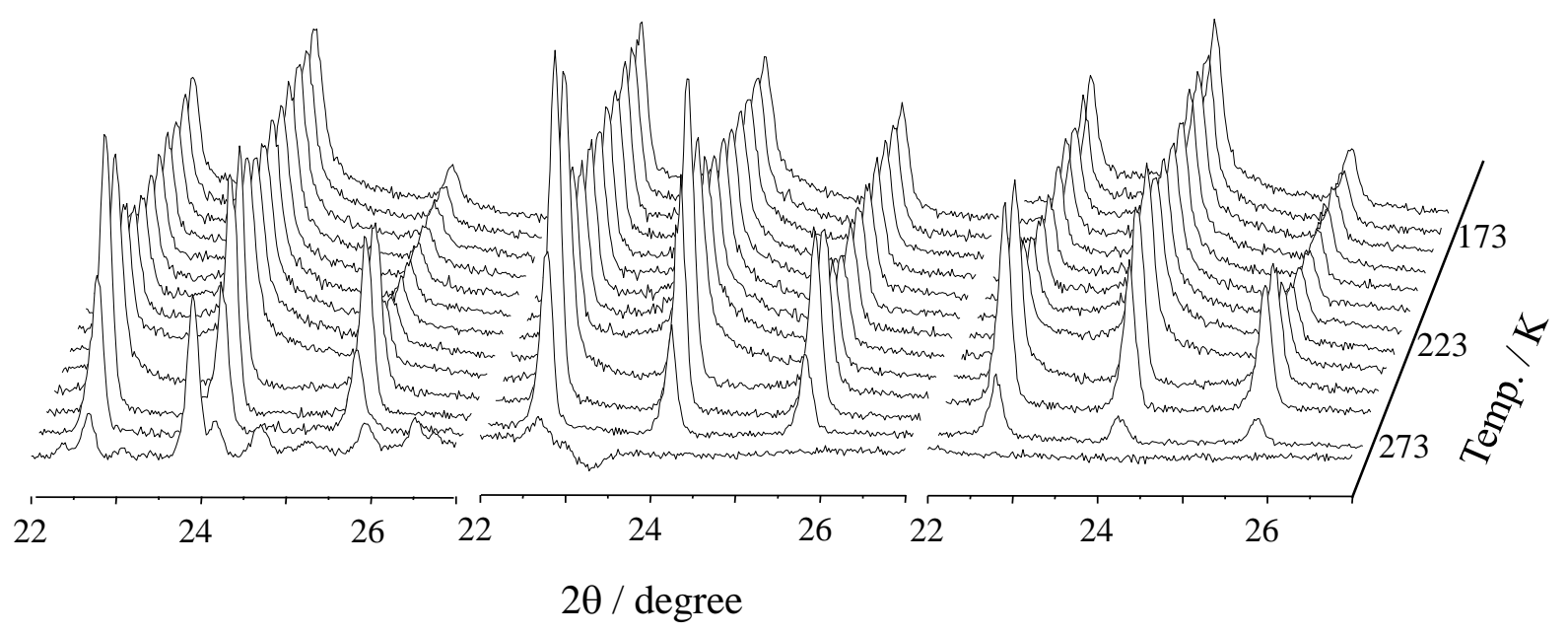

Fig. 5 


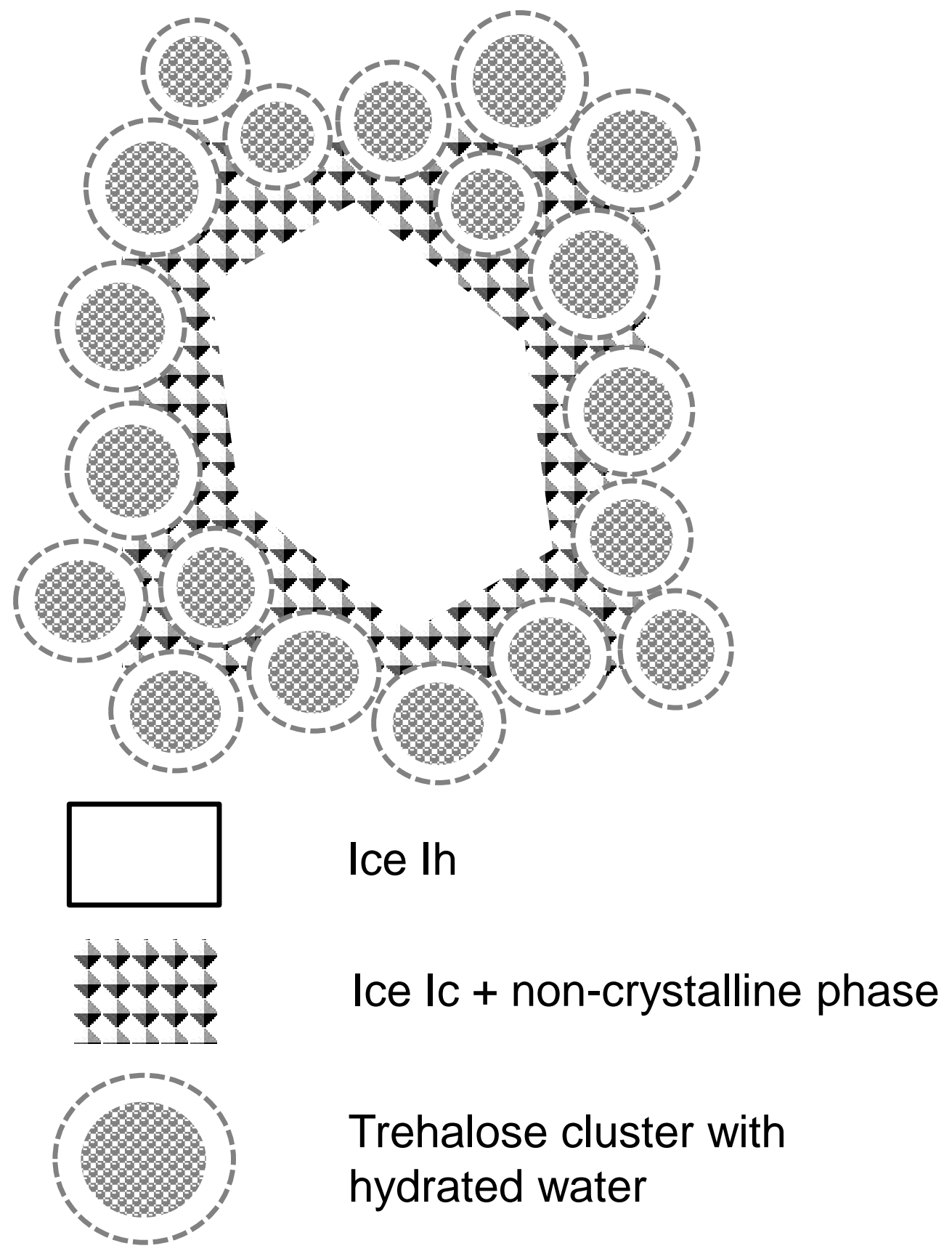

Fig. 6 


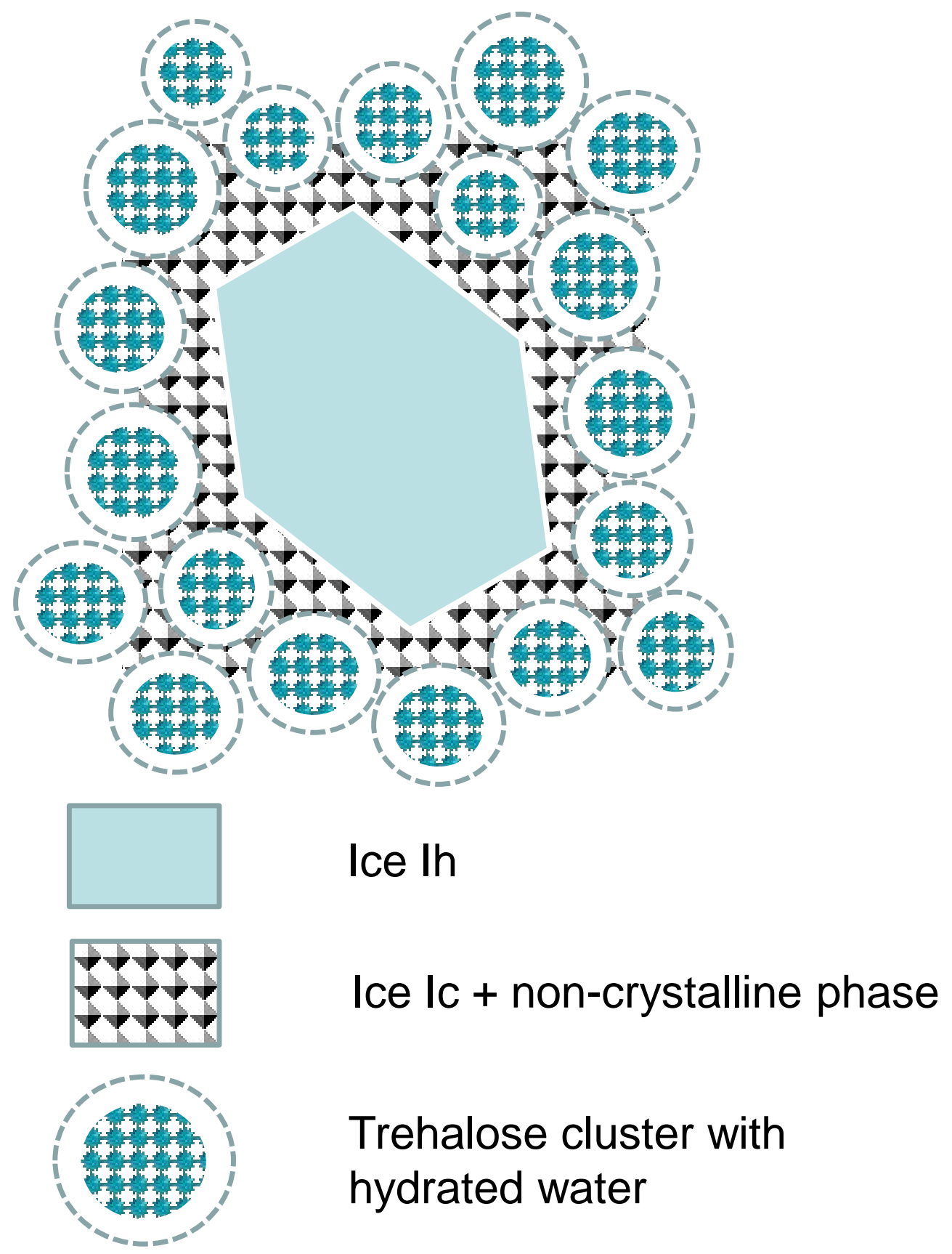

Graphic contents entry 